\title{
EL XIII CONGRESO DEL PIT CNT “WLADIMIR TURIANSKY”: UNA OBSERVACIÓN ETNOGRÁFICA.
}

\author{
The XIII Union Congress of the PIT CNT: "Wladimir Turiansky": an \\ ethnographic observational approach.
}

Marcos Supervielle'

Leonel Rivero²

Leonardo Cosse ${ }^{3}$

\section{Resumen}

Los congresos constituyen la máxima instancia de autoridad de los sindicatos, pero a su vez cumplen otras funciones, y ofrecen una condensación de elementos que ayudan a conocer aspectos culturales que muchas veces aparecen esquivos al análisis. En este sentido, la observación etnográfica de los congresos, tal como lo plantean Béroud y Denis (2015), ofrece una herramienta propicia para captarlos e incorporarlos al análisis. Sin embargo para poder aproximarse como observador a este tipo de instancias, es necesario hacer explícitas ciertas categorías de entendimiento previas, que el sociólogo construye a partir de las que utilizan los propios actores involucrados. En esta oportunidad, en el marco del proyecto El sindicalismo uruguayo en el proceso de construcción de una Cultura del Trabajo para el Desarrollo ${ }^{4}$, se desarrolló la observación del XIII Congreso del Plenario Intersindical de Trabajadores Convención Nacional de Trabajadores ${ }^{5}$ (PIT-CNT) de Uruguay. En este artículo se desarrollan las categorías de entendimiento con las que nos acercamos al fenómeno, así como los principales resultados de la observación.

Palabras clave Movimiento sindical; etnografía; categorías de entendimiento.

\begin{abstract}
The trade union congresses constitute the highest instance of authority of the trade unions, but at the same time fulfill other functions, and offer a condensation of elements that help to understand cultural aspects of trade unionism that often appear elusive to the analysis. In this sense, the ethnographic observation of the congresses, as proposed by Béroud and Denis (2015), offers a useful tool to capture and incorporate them into the analysis. However, in order to be able to approach these types of instances as an observer, it is necessary to make explicit certain

\footnotetext{
1 Profesor de sociología de la Universidad de la República, Montevideo, Uruguay. Email: msupervielle@gmail.com.

2 Profesor en Facultad de Ciencias Sociales de la Universidad de la República, Montevideo, Uruguay. Email: 1riverocancela@gmail.com

3 Universidad de la República, Montevideo, Uruguay. E-mail: leonardocosse@gmail.com

4 Proyecto financiado por el Programa de Investigación y desarrollo de la Comisión Sectorial de Investigación Científica de la Universidad de la República (CSIC-UdelaR)

5 El PIT-CNT es la principal central sindical de trabajadores en el Uruguay.
} 
categories of prior understanding, which sociologist constructs based on the categories of understanding of the actors involved. On this occasion, within the framework of the project "Uruguayan Trade Unionism in the process of building a Culture of Work for Development" (R + D CSIC, 2017-2018), the observation of the XIII Congress of the PIT-CNT of Uruguay was developed. In this article we present the categories of understanding with which we approach the phenomenon, as well as the main results of the observation.

Keywords Trade union movement; ethnography; categories of understanding.

\section{Introducción}

Los autores de este trabajo formamos parte de un equipo de investigación que lleva adelante el proyecto $E l$ sindicalismo uruguayo en el proceso de construcción de una Cultura del Trabajo para el Desarrollo (CSIC-UdelaR, 2017-2018) dirigido por Mariela Quiñones desde el Departamento de Sociología de la Facultad de Ciencias Sociales de la Universidad de la República.

En el curso de esta investigación, tuvo lugar el XIII Congreso del PIT $\mathrm{CNT}^{6}$, instancia que entendimos como una excelente oportunidad para incorporar una importante cantidad de información, así como chequear la validez de hipótesis que comenzaban a surgir en el proceso de investigación. La propia dinámica del congreso ofrecía además una oportunidad para construir otro tipo de dato, y de acceder a información a la que no se llega a través de entrevistas en profundidad.

Tomamos al Congreso básicamente como un "analizador" (LOURAU, 1991), es decir, como una manifestación de la cultura sindical, y como veremos más adelante, de la propia cultura de trabajo de los trabajadores. Entendemos por cultura del trabajo, una trama de significados compartidos en torno al mundo del trabajo (esto es tanto a nivel organizacional, de la relaciones de producción, como de las relación del trabajador con su trabajo).

Partimos de la hipótesis que la cultura sindical es una manifestación de la cultura de trabajo. En la medida en que el

\footnotetext{
6 El Plenario Intersindical de Trabajadores / Convención Nacional de Trabajadores (PIT-CNT) es la única central sindical en el Uruguay. La gran mayoría de los sindicatos en el Uruguay forma parte de esta central. El Congreso es la autoridad máxima del PIT-CNT y se reúne de forma ordinaria cada 2 años.
} 
El XIII Congreso del PIT CNT "Wladimir Turiansky" | Marcos Supervielle, Leonel Rivero \& Leonardo Cosse

sindicalismo es una actividad intrínsecamente ligada al ámbito del trabajo, podemos decir que toma sus supuestos básicos de las culturas del trabajo, sobre todo aquellos elementos que surgen de la dinámica de las relaciones entre capital y trabajo, para la cual el sindicalismo cobra sentido ${ }^{7}$.

Por otro lado, este enfoque metodológico que propone mirar la cultura de trabajo a partir de la cultura sindical nos permite salir de las perspectivas más clásicas del sindicalismo, es decir, de aquellas centradas en las dinámicas de poder que funcionan a la interna del movimiento sindical, en las tendencias existentes y en los acuerdos que se logran -la búsqueda de la unidad, por ejemplo-; o bien, de la mirada del sindicalismo como un actor pleno, que obviando sus tensiones y clivajes internos, juega un papel central en los procesos de redistribución económica, con potencial político cuando se transforma en un actor en este campo ${ }^{8}$.

Nuestra hipótesis es que el sindicalismo, y más concretamente, la cultura sindical, es de alguna manera una manifestación de la cultura del trabajo de los propios trabajadores. Es decir, hay continuidad en ambas. Esto no implica entender la cultura del trabajo como equivalente de cultura sindical. En efecto, la esfera de la cultura del trabajador con respecto a la labor que realiza, y la esfera politica de organización sindical tienen sus dinámicas propias. Sin embargo, según aquí entendemos, a través de las reivindicaciones sindicales se

\footnotetext{
$7 \mathrm{Si}$ no fuese así, sería simplemente un movimiento político con una plataforma independiente del ámbito del trabajo. En este caso el sindicalismo tendría su "ideología propia" por fuera de la de los trabajadores. Más allá de reconocer la autonomía relativa de la dinámica política al interior de la lucha por el poder (a la que nos referiremos también) y de la lucha por la hegemonía ideológica, muy rápidamente estas estructuras sindicales o bien se transformarian en una suerte de burocracia sindical, con intereses propios, desligados de los intereses autóctonos de los trabajadores, o bien tendería a desaparecer ya que se haría cada vez menos representativa, llevando a que los trabajadores busquen otras formas de hacer que se oigan sus voces. Cualquiera de estos dos fenómenos existe en muchos países del mundo, pero claramente no representan el "corpus central" del sindicalismo uruguayo. Al respecto hay mucha evidencia pero no nos detendremos en ella, más que señalar que, en una tendencia contraria a la internacional, el sindicalismo uruguayo se encuentra en un período de fuerte expansión de su afiliación, alcanzando a 400.000 trabajadores $25 \%$ de la PEA.

8 Estas son las dos miradas académicas clásicas latinoamericanas sobre el sindicalismo (ABRAMO y MONTERO, 2000) que vuelve a surgir hoy en día con los eventos actuales en Brasil (huelga de transportes y de petróleo), y que se coloca en un plano similar en Argentina con las políticas de ajuste.
} 
evidencian pautas importantes acerca de las representaciones y significados sobre el trabajo.

Al mismo tiempo, se parte de la base de que la observación etnográfica, como técnica, permite abordar el fenómeno cultural a partir de las dinámicas tácitas que se generan en su marco. Es de remarcar que el Congreso es la instancia de máxima decisión del movimiento sindical, de modo que lo que alli se expresa tiene un fuerte valor performativo y configurativo, en tanto los lineamiento políticos y las autoridades electas derivan de lo allí acordado.

Desde nuestra perspectiva, la mejor manera de observar un evento colectivo singular, es decir, no rutinizado, como es el caso de un Congreso, es de observarlo de forma etnográfica. Para esto es necesario:

a) Contar con categorias de entendimiento (SUPERVIELLE, 2017) previas para darle significado a los distintos sucesos que pasan delante de nuestra vista. En el artículo referido pasamos revista de las principales teorias que utilizan las categorias del entendimiento como conceptos relevantes en sus sistemas conceptuales. En este trabajo intentamos darle un sentido operativo a las categorias del entendimiento. Estas, previamente construidas -en tanto que sociólogos- a partir de un conocimiento sólido del campo en donde se inscribe el evento que observamos, nos permite dar profundidad a nuestras observaciones puntuales. También permite incorporarlas a un sistema interpretativo, sea dándole mayor consistencia a hipótesis previamente elaboradas, sea modificando estas hipótesis o ampliando su proyección para dar cuenta del fenómeno que se intenta abordar. En la propia observación, nos basamos en algunos eventos o aspectos que nos sorprendieron. Esto nos enfrentó a que nuestras categorías del entendimiento construidas previamente no correspondian totalmente a las categorias del entendimiento de los sindicalistas y ello nos llevaba a tener que interpretar aquello que nos había sorprendido, reprocesar información y cotejar su coherencia con 
El XIII Congreso del PIT CNT "Wladimir Turiansky" | Marcos Supervielle, Leonel Rivero \& Leonardo Cosse

las interpretaciones nuevas que proponemos, permitiéndonos avanzar así sobre el conocimiento de las características de la cultura sindical. A su vez, el tomar en consideración la perspectiva del otro es una de las especificidades epistemológicas de la investigación etnográfica (BECKER, 1991)

b) Tener una grilla de observación que nos permita ordenar una serie de aspectos a considerar, que son necesarios para realizar una observación relativamente completa de un evento colectivo, sin dejarnos llevar por algún aspecto que nos impresiona puntualmente. A su vez permite recoger información de tipo emotivo que no puede ser capturada si no es a través de la observación directa de los participantes de los participantes del Congreso. Observar las interrelaciones que se dan entre diferentes planos, racionales y afectivos, nos permite comprender mejor lo que sucede en el Congreso como fenómeno colectivo. El nivel concreto de comprensión que se puede alcanzar a través de la observación etnográfica no es alcanzable desde la perspectiva más abstracta de la búsqueda de correlaciones entre variables. Esta también es una especificidad epistemológica de las investigaciones basadas en observaciones etnográficas. (BECKER, 1991).

\section{Las categorías de entendimiento.}

En el siguiente apartado señalamos las principales categorias de entendimiento que fueron surgiendo de la investigación en curso sobre la de cultura de trabajo en el sindicalismo uruguayo.

Nuestro aporte y su originalidad, consiste en observar desde otra perspectiva que consideramos que es necesario incorporar tanto en los estudios del trabajo como en los estudios del sindicalismo. Perspectiva que supone un mayor distanciamiento de la casuística del momento, aunque sin abandonar la evidencia empírica como soporte de razonamientos y hallazgos. Lo que se busca es comprender las 
potencialidades del trabajo para el desarrollo, pero también para dar desde una mirada crítica de mayor profundidad la politica de desarrollo que viene impulsando el Frente Amplio en sus gobiernos. La perspectiva que proponemos parte justamente de dar relevancia a la cultura de trabajo como eje central para comprender el comportamiento del actor sindical.

Esto nos lleva a buscar resolver un problema teórico bien complejo, ¿Cómo es posible descifrar las características de la cultura de trabajo existente a partir de las manifestaciones que se dan a nivel sindical, ya sea en el Congreso o en las entrevistas que realizamos a dirigentes sindicales?

Creemos que para hacerlo debemos partir de tres diferentes categorías del entendimiento sobre el trabajo:

- Las orientaciones de los trabajadores para trabajar.

- Las interacciones de "clase” en los ámbitos de trabajo.

- La elaboración de programas socio económicos alternativos a los que lleva adelante el gobierno.

Es evidente que estos niveles no son totalmente autónomos y es claro que se contaminan entre sí, sin embargo su existencia es real y también su desagregación permite profundizar un análisis de las conductas complejas, a veces aparentemente contradictorias que adoptan los trabajadores a través de los sindicatos para manifestarse públicamente.

\section{Las orientaciones de los trabajadores para trabajar}

Tomando la propuesta de Meda y Vendramin, se puede hablar de dos orientaciones genéricas hacia el trabajo por parte de los trabajadores: pragmática (o instrumental) y expresiva (MEDA y VENDRAMIN, 2013).

La orientación de tipo pragmático (instrumental) es aquella en que se privilegia entre los trabajadores una visión del trabajo como una constricción que puede ser: i) vivida positivamente, porque permite 
El XIII Congreso del PIT CNT "Wladimir Turiansky" | Marcos Supervielle, Leonel Rivero \& Leonardo Cosse

realizar otras actividades que despierte más interés (pero que quizás no genera suficientes ingresos para vivir); b) el trabajo entendido como un medio para ganar dinero; el trabajo se vuelve entonces una suerte de fuente de remuneración cuyo destino es el consumo.

En nuestras entrevistas percibimos que en muchos sindicatos que agrupan trabajadores administrativos, o trabajadores de actividades comerciales y de servicios, tienen esta orientación de manera dominante; aunque lo hemos encontrado en otros rubros, tales como la industria química $\mathrm{y}$, desde luego, es extensible a diversas ocupaciones.

La segunda orientación es la de tipo expresivo que reagrupa fundamentalmente dos grandes contingentes: i) aquellos para quien el trabajo aparece como el soporte de su desarrollo personal; y ii) aquellos para quienes el trabajo es central en la conformación de su identidad tanto personal como sobre todo pública. Esta segunda orientación es dominante en dos grandes campos: el campo de los trabajos orientados a lo social (de asistencia o promoción social, educación y salud, entre otros), o aquellos orientados a actividades creativas (artísticas, de innovación, de investigación, pero también puede ser aquellos trabajos que tienen contenido artesanal)

Estas dos orientaciones son fuente de dos aproximaciones muy distintas al sindicalismo y ello se traslada a sus plataformas y el tipo de reivindicaciones dominantes.

La primera (pragmática) cobra fuerza no tanto en el campo de la orientación al trabajo en cuanto tal, sino que en el de la defensa de derechos que se consideran fundamentales por parte de los trabajadores del sector. Esto comienza por el propio reconocimiento de los trabajadores como ciudadanos con derechos, que en la práctica se considera que se niegan por parte de los dirigentes de las empresas en las que trabajan. Y ello va desde el propio reconocimiento del sindicato, hasta otros campos más específicos, como son el salario, las condiciones de seguridad y salud en el trabajo, tanto en el plano de los 
derechos colectivos, como también a nivel individual. Muchos trabajadores se vinculan con el sindicato exclusivamente para que este los ayude a resolver problemas individuales, a través de los servicios jurídicos o de la intervención directa del delegado sindical en el ámbito de diálogo con la dirección de la empresa. Este tipo de orientación al trabajo, por sus características específicas no predomina en las manifestaciones colectivas como fue el caso del Congreso.

La segunda orientación (expresiva) genera otras connotaciones y sí se expresa muy fuertemente en el Congreso, justamente porque al tener incidencia a nivel de los sindicatos de ciertos sectores (social, educativo, salud, etc.) genera proyectos globales de cómo debería funcionar el sector, anteponiéndolos a proyectos existentes impulsados por el gobierno o desde los partidos politicos. Y por otro lado, genera visiones de cómo debería ser la participación de los trabajadores en la definición de las grandes orientaciones del sector. Por lo general, en mayor o en menor medida, los trabajadores de estos sectores se sienten excluidos de las decisiones estratégicas que se toman en sus sectores, en la organización de los mismos (en lo atinente a la organización de sus unidades productivas o servicios), o en los protocolos para la incorporación de tecnología que modifique los equilibrios de poder existente y también las condiciones de trabajo. Es casi natural que en estos sectores en donde el trabajo representa un papel central en la vida de los trabajadores (no solamente la función remuneradora), que sea un campo de proyectos, movidos por un deber ser que siempre entra en conflicto con lo que es. En segunda instancia y justamente por la misma razón, es en estos sectores en donde más hay conflicto de proyectos al interior del propio sindicato Ello muchas veces se cristaliza a través de agrupaciones al interior del sindicato. También como proyección de su que hacer laboral los sindicatos con esta orientación dominante han sido muy activos en cuanto a las reivindicaciones vinculadas a los cambios de modelo civilizatorio (incorporando demandas de género, generaciones, discapacidad, medio ambiente, etc.) 
El XIII Congreso del PIT CNT "Wladimir Turiansky" | Marcos Supervielle, Leonel Rivero \& Leonardo Cosse

$\mathrm{y}$ en la ampliación de las alianzas del sindicalismo con otros movimientos sociales.

Como digresión nos interesaría señalar que "la cultura de trabajo para el desarrollo" 9 sea por parte del Estado como por parte de los dirigentes de las empresas (privadas y públicas) tendría como soporte central, la orientación expresiva en el trabajo. A nivel del gobierno porque es esta la que permite pensar, negociar, y direccionar el trabajo para el desarrollo. Y en el sector empresarial porque se busca que el trabajador se oriente a ser más creativo y más responsable en las nuevas funciones en el trabajo y abandone la matriz cultural del trabajo repetitivo de tareas estándar. Paradójicamente, es justamente a este nivel que el trabajo expresivo aparece como más contestatario y como más exigente de las responsabilidades de los dirigentes, sean de empresas públicas o privadas. A su vez, daría la impresión de que las empresas privadas y también las públicas y las administraciones, prefieren ver al sindicato únicamente como "reclamador" de aumento de salarios y que no se inmiscuya con las opciones ideológicas que le dan sustento a decisiones programáticas o estratégicas en las instituciones.

\section{Las interacciones de "clase" en los ámbitos de trabajo}

A la orientación hacia el trabajo de los trabajadores, se le suma la relación de clase. Relación que construyen de forma cotidiana con las autoridades que tienen en los ámbitos concretos en donde trabajan. Esta cultura de trabajo se objetiva en un conjunto de reglas concretas, explícitas y tácitas del trabajo.

En efecto, en la medida en que los trabajadores saben de su condición de subalternidad al ser asalariados, la conformación de reglas de control explícitas le son necesarias para saber qué expectativas tienen sobre cómo hacer el trabajo que se les pide, y con ello acotar la arbitrariedad de las evaluaciones que se realizan de su trabajo. Las

\footnotetext{
9 La "cultura del trabajo para el desarrollo" representa la directriz estratégica del Ministerio de Trabajo y Seguridad Social para el periodo 2015-2020, plasmada en un documento que lleva el mismo nombre.
} 
reglas tácitas hacen al real funcionamiento de las organizaciones complejas. Las normas explícitas son del dominio del campo jurídico. Las reglas tácitas forman parte del campo de la confianza, este es, el campo de las conductas esperadas de los actores, que participan en el mundo de trabajo concreto. Este campo obviamente remite a las conductas individuales pero el nivel que nos interesa es el de las conductas colectivas y particularmente en las que interviene el sindicato.

Tanto las reglas explícitas como las tácitas están en continua redefinición. Las primeras a partir de exigencias de mayores derechos o de mayores deberes. Con ello forman parte de la negociación permanente de los sindicatos con sus mandantes. Las segundas, exigen formas más sutiles de cambio ya que se inscriben en la apreciación del alter, y corresponden a las lealtades y a las reglas que todos suponen que existen. $\mathrm{Si}$ se realizan acciones oportunistas, con la que se consiguen "ventajas" transigiendo estas reglas, el gran costo es una falta de confianza generalizada, que trasciende la acción oportunista puntual repercutiendo en todas las relaciones entre el sindicato y sus mandantes. En este sentido el "costo de transacción" derivado de un cambio realizado por los mandantes, tiene consecuencias de largo plazo, por la falta de confianza en ellos por parte de los sindicatos. Y este fue, sin lugar a duda una de las fuentes de la postura que adoptaron muchos sindicatos con respecto a los dirigentes de empresas públicas o privadas o de administraciones públicas y fueron la fuente de las posiciones que se expresaron en el Congreso.

De hecho, posturas de este tipo no son novedosas en el sindicalismo uruguayo. Si se considera la década de los años sesenta, se encuentra una continua referencia a empresarios y patrones privados que eran considerados como el prototipo del empresario capitalista, al que no se le podía tener confianza en ningún plano.

Esto último no sucede más en el sector privado, o al menos no se hizo mención en el Congreso. Las crisis de confianza y las 
El XIII Congreso del PIT CNT "Wladimir Turiansky" | Marcos Supervielle, Leonel Rivero \& Leonardo Cosse

manifestaciones de indignación que aparecieron en el Congreso fueron en las empresas públicas, como en ferrocarriles, y en administraciones como la intendencia de Montevideo o con respecto a la salud pública. En la mayoría de los casos, se hizo mención explícita de los nombres de los que ocupaban las cúspides de las administraciones. Todas administraciones que están dirigidas por personas designadas o electas por el Frente Amplio.

Pareceria ser que los distintos procesos de cambio que se han implementado en empresas públicas y administraciones no se han realizado intentando preservar la confianza de los trabajadores en general, y en particular de los sindicatos que los representan en las Administraciones, en el buscar atenerse a las reglas de juego establecidas. Consideramos que el nivel de indignación que se ha generado tiene además el agravante que estas cúspides de la administración en cuestión fuesen justamente del Frente Amplio. En principio, porque deberian estar orientadas por la voluntad de fortalecer la dignidad de los trabajadores; cuando, por lo contrario, lo que han hecho es menospreciarlos, o establecer normas que reducen la libertad de acción y de protesta de los trabajadores.

\section{La elaboración de programas socio económicos alternativos a los que lleva adelante el gobierno}

En el Congreso se propusieron tres documentos como base programática, rompiendo la tradición de presentar un documento único. Es tradición del sindicalismo uruguayo elaborar sus propios modelos de desarrollo social y económico. Estos modelos le han servido históricamente al PIT-CNT como mecanismo para evaluar las decisiones económicas o sociales que toma el gobierno, de forma sistemática, partiendo de la perspectiva de los intereses globales de la clase trabajadora. También le permite al PIT CNT tener un marco de referencia global para orientar su acción estratégica y táctica sindical general. La votación de los tres documentos presentados, aunque no era 
lo deseado, permitió legitimar la orientación del sindicalismo hasta el próximo Congreso.

En los tres documentos se expresan básicamente dos corrientes, que a los efectos de esta discusión pueden resumirse de esta forma: i) una vertiente que se autoproclama "independiente" del gobierno, que lo coloca en el lugar de alteridad absoluta "sea del partido que sea", y ubicándolo como un enemigo de clase intrínseco al movimiento sindical. Desde esta retórica, se expresa que los signos progresistas del partido de gobierno esconden procesos de dependencia con el capital, extranjerización de la tierra, y aplicación de medidas de precarización laboral, privatización, y represión del movimiento sindical; ii) una vertiente que se expresa como "independiente pero no indiferente", y que lee la coyuntura económica de forma favorable, la opone retóricamente a los años de pos-dictadura y la década del '90 caracterizada por su fuerte signo neoliberal-, remarca los espacios de negociación instalados, el diálogo - encarnado a través de diversas figuras del sindicalismo-, la mejora del salario real, y el crecimiento económico. Esto sin desmerecer una serie de elementos críticos hacia el gobierno y sus políticas.

Esta dinámica se vuelve asequible en las diferentes oratorias, y se plasma en los documentos que los sindicatos elevan a consideración del Congreso. Asimismo, los consensos y negociaciones se ven reflejadas en las combinaciones de sindicatos que firman dichos documentos, permitiendo comprender en poco tiempo las ordenaciones políticas a la interna del movimiento sindical. Es así que la observación del congreso se transforma en una técnica potente que permite entender las formas en que se articulan las diferentes corrientes sindicales, y el contenido que le dan a las reivindicaciones.

La unidad es, sin lugar a dudas, el concepto central que dominó las intervenciones del Congreso. Que esto sea así, que la unidad no sea disputada o cuestionada, habla de un proceso de construcción de legitimidad de las estructuras sindicales cuyo peso es necesario valorar. 
El XIII Congreso del PIT CNT "Wladimir Turiansky" | Marcos Supervielle, Leonel Rivero \& Leonardo Cosse

Más interesante aún resulta indagar las discusiones que se dan en el marco de la unidad, así como las formas en las que la unidad se construye y se sostiene.

En primer lugar, podemos apreciar que existen una serie de temáticas y discusiones que se colocan sobre la mesa y generan adhesiones indisputadas en el movimiento sindical. La primera y más evidente de ellas refiere a las reivindicaciones vinculadas a la discapacidad, plasmadas en el reclamo de una Ley de Empleo para Personas con Discapacidad, que es parte de la agenda del movimiento sindical en los últimos años. Dicha demanda se ha consolidado en el sentido común sindical como un elemento de justicia social básico, que no ofrece discusiones, sino que su inclusión en la plataforma sindical genera importantes consensos.

En segundo lugar, tras haber ganado centralidad en la esfera sindical y social, podemos ubicar la cuestión de género. La misma tiene antecedentes fuertes dentro del movimiento, vinculado por ejemplo, a la constitución de una Secretaría de Género (rango máximo dentro del organigrama del PIT-CNT), la inclusión de cláusulas de género en la mayoría de los convenios emanados del Consejo de Salarios, y que se cristaliza en este Congreso en la exigencia de una mayor integración de mujeres al Secretariado Ejecutivo del PIT-CNT. En tal sentido, la votación final por la cual se incluyen cinco mujeres en este ámbito, responde a este proceso acumulativo que ha tenido el sindicalismo en materia de género, y que incluye la construcción a la interna del movimiento sindical, y a la externa, a partir de la Intersocial feminista. Es así que la cuestión de género se convierte en un aspecto central, que disputa el modelo civilizatorio, y al trabajador varón como el "sujeto de la historia".

\section{La observación etnográfica}

En el artículo Les Congrès syndicaux: un objet d'étude sociologique? (BÉROUD \& DENIS, 2015) sostienen la idea de que la 
consideración de los congresos sindicales en tanto forma social (en el sentido de Simmel), aporta elementos útiles y una perspectiva valiosa para comprender mejor a los movimientos sindicales. Esto implica considerar la forma congreso sindical en cuanto tal, pero a la vez cómo ésta sirve para vehiculizar ciertos contenidos. Desde este punto de vista, en el artículo se sostiene que los congresos pueden aportar información en cuanto al equilibrio de las fuerzas internas y la salud de la organización; la composición sociológica y politica de los representantes y de los dirigentes; y la renovación de sus miembros y de su apertura. (BÉROUD y DENIS, 2015)

Como forma de contribuir a orientar la observación por parte del investigador etnográfico, se desarrollan una serie de funciones de las que es posible dar cuenta, y que están presentes en todos los congresos sindicales: retórica; educativa; demostrativa; deliberativa; legislativa; electiva; y festiva. Tomando como guía estas funciones, se intentará aquí dar cuenta de algunos elementos relevantes observados en el congreso del PIT CNT.

\section{Función retórica (o expresiva).}

Esta función resulta una de las más evidentes de los congresos, y hace referencia a la toma de la palabra de los sindicalistas a través de la representación de su sindicato de base, e incluso -como se pudo ver en el Congreso del PIT CNT- de la representación de sectores minoritarios del mismo.

En relación a esta función uno de los elementos que destaca del congreso observado tiene que ver con la cantidad de sindicalistas que pide la palabra para poder hablar y hacerse oír; tanto en la instancia de las comisiones como de plenario final. Incluso es posible observar que demandan la palabra varios representantes del mismo sindicato. Esto da cuenta de la convicción por parte de los oradores de que hay algo para decir, de que es necesario expresarlo, y de que el congreso es el lugar para hacerlo. 
El XIII Congreso del PIT CNT "Wladimir Turiansky" | Marcos Supervielle, Leonel Rivero \& Leonardo Cosse

Particularmente en la inauguración del congreso, en el que toma la palabra el presidente del PIT CNT, se recurre al ubi sunt como forma retórica, es decir, a la memoria de aquellos que ya no están y que fueron figuras trascendentes en la construcción del movimiento sindical uruguayo. Esto contribuye a construir legitimidad a partir de la continuidad de la historia del movimiento sindical. Incluso en la misma inauguración se invita a ex dirigentes sindicales, como forma de reconocimiento, de dar continuidad a ciertas nociones que conforman la identidad del movimiento, como lo es la idea de unidad.

Determinados por la forma congreso, los discursos presentan cierto tamiz particular, que se puede explicar por la intención de conmover al auditorio, así como por el poco tiempo con el que cuentan los oradores ( 5 minutos). Por eso se observa que muchos recurren a frases contundentes como: "independientes pero no indiferentes", en referencia al gobierno de turno; o "que los partidos busquen sus votos solos", con un sentido similar al anterior, remarcando la independencia en relación al sistema político; o "trataron de dividirnos", en referencia a actores externos, principalmente al sector empresarial, que se entiende que conspira contra la unidad o celebra la fragmentación del movimiento sindical.

La retórica, además, se desarrolla en dos vertientes de gran importancia: por un lado, una poética que busca conmover a los oyentes, ("si puedo salir abrazado con el compañero, piense lo que piense, estaremos construyendo la unidad en la diversidad") por otro lado, una política, que busca trazar un mapa de comprensión del estado de situación actual ("el neoliberalismo no va a pasar si encuentra a los trabajadores organizados").

II. Función educativa.

La función educativa hace referencia a la importancia de los congresos en la socialización de los jóvenes militantes a los usos y costumbres de la organización. 
Esta función se da por la vía de los hechos, por la propia participación de sindicalistas jóvenes, pero a su vez depende de las características del proceso de recambio generacional. En este sentido, se evidenció la presencia de muchos delegados para los cuales era el primer congreso (algo menos de la mitad según un sondeo espontáneo realizado en una de las instancias del congreso)

La educación sindical, se genera en un sentido amplio, tanto desde las generaciones mayores hacia las más jóvenes, como al interior de las generaciones: a través de la evocación de los principales referentes que marcan la trayectoria sindical; a través de la integración de la problemática laboral en un enclave macropolitico; y a través de las prácticas de funcionamiento mismo del congreso (la entrega de los estatutos, la votación de pautas de funcionamiento, la elaboración de informes, la entregar el material, entre otras)

\section{Función demostrativa}

El congreso es un momento en el cual se comunica hacia la interna como hacia el exterior, y se popularizan las principales orientaciones y decisiones del movimiento sindical.

En este marco, se destaca el reunirse más de dos mil personas (mil de ellos delegados ${ }^{10}$ representantes designados de sus sindicatos de base), con muy alta asistencia, inclusive de delegados suplentes.

Por otra parte, el Congreso está lleno de demostraciones de fuerza del movimiento sindical. En la inauguración, se invita a los políticos, a delegados extranjeros, se hace una demostración de permanencia en el tiempo más allá de los gobiernos, utilizando todos los recursos disponibles: la oratoria, el teatro, el audiovisual, la música. Es de destacar que se llenan espacios masivos, tales como el local de la central sindical, y estadios deportivos.

En relación a la función demostrativa a la interna del movimiento sindical, el momento de las votaciones parece clave, sobre todo cuando 
El XIII Congreso del PIT CNT "Wladimir Turiansky" | Marcos Supervielle, Leonel Rivero \& Leonardo Cosse

las resoluciones se toman por unanimidad, por el sentimiento de unidad que transmite el acuerdo entre la totalidad de los congresistas. La forma en que se vota -mediante el gesto de levantar la mano-, la materialidad y corporalidad de la expresión, da cuenta también de la importancia de la función expresiva, que se manifiesta a la vista del otro y contribuye a acrecentar el sentimiento de pertenencia al colectivo. Finalmente los aplausos después de los votos, celebran las decisiones que se toman, pero más aún la forma en que se toman y el hecho mismo de decidir en colectivo.

\section{Función deliberativa}

En el congreso se colocan en discusión las principales temáticas que afectan al sindicalismo uruguayo. Esto se expresa en un gran número de documentos base e informes de comisiones que buscan dar una mirada integrada en torno al posicionamiento sindical, no sólo en relación al mundo del trabajo, sino al escenario político y social nacional e internacional.

En tal sentido, la discusión se realiza en torno a una agenda de temas y apuestas mayores que involucran al sindicato: el tipo de trabajo, los modelos de desarrollo, el vínculo del movimiento sindical con el movimiento social, las relaciones con el gobierno, los modelos democráticos, las alianzas nacionales e internacionales, el uso de la academia, entre otros, que buscan poner a discusión un mapeo cognitivo, un discurso afirmativo del mundo.

En relación a la dinámica de discusión, se observa la apertura a que todas las posiciones se escuchen, que las expresiones de desconfianza sean debatidas con argumentos, y que todos los documentos sean valorados y aprobados, en mayoría o minoría. En el plano organizativo de la discusión uno puede percibir que, bajo el aparente caos en el cual se desarrolla la sesión, todos los oradores se sujetaron al tiempo que se les había concedido. En este sentido no hubo advertencias de la mesa, y además se habló en casi todas las ocasiones 
con un gran respeto y tolerancia de las posiciones distintas a las que se defendian.

\section{Función legislativa}

La función legislativa es la base misma del Congreso, que tiene como objetivo discutir y votar los textos que le son sometidos por la dirección saliente con respecto a su actividad pasada (balance económico, balance social) y los proyectos de futuro.

Más allá de esto, la función legislativa buscó sustentarse sobre la electiva, privilegiando la búsqueda de unidad. Por tales motivos, se destaca la aprobación de todos los documentos, así como la votación por consenso de los representantes, dejando en manos de la Mesa Representativa las propuestas realizadas.

Asimismo, se destaca el respeto cívico a las normas de funcionamiento, de modo que, al darse los resultados, nadie protesta ni se queja de la votación, lo que habla de la legitimidad de las formas de decisión establecidas. Pero también en la medida que los que tuvieron mayoría en la presentación de un documento no festejaron su victoria. Se percibe también un cierto sentimiento de frustración por el no haber podido alcanzar un documento único negociado entre las distintas posiciones, que llevó a la presentación de tres documentos por separado. Este sentimiento de frustración, parece luego superarse con la votación de listas únicas.

\section{Función electiva}

Esta función podría decirse que es el corazón del congreso, en tanto el mismo se reúne para votar a sus representantes y a la la comisión directiva. En tal sentido, a lo largo del congreso se visibiliza una fuerte discusión en torno a los espacios de conducción del movimiento sindical, su naturaleza y jerarquía. Así, se discute sobre qué cantidad de miembros debe tener la mesa representativa, qué roles deben atribuirse al Secretariado Ejecutivo, y cuantas Secretarias y Departamentos deben consolidarse. 
El XIII Congreso del PIT CNT "Wladimir Turiansky" | Marcos Supervielle, Leonel Rivero \& Leonardo Cosse

Sin embargo, en relación a la Mesa Representativa y el Secretariado Ejecutivo, órganos máximos de conducción del movimiento sindical (subordinados al Congreso), la tradición es presentar una lista única. En tal sentido, los resultados muestran que fue posible hacerlo, lo que redunda en una consolidación del movimiento sindical único, característica particular uruguaya.

\section{Función festiva}

La función festiva se hace presente porque el congreso es una instancia de reencuentros y de ágapes casi religiosos entre militantes. La misma fue plasmada en las diversas instancias que realizadas en el marco del mismo. En la inauguración, que supone un reencuentro de sindicalistas de muchos sindicatos. En el festejo sin incidentes, revalorizando la democracia sindical, que se consolida culturalmente en tanto el colectivo se sienta representado, más que por la formalidad de los procedimientos. Asimismo, se realizan rituales que dan sentido de pertenencia e historicidad al movimiento sindical, plasmado en los cantos como el Himno de la Internacional, y otros como el grito de "Unidad, CNT".

\section{Conclusiones}

En el XII Congreso del PIT-CNT se puso en evidencia la fuerza de la noción de unidad a través de cierta confluencia entre aquellas posturas que derivan de una "cultura de trabajo" de tipo expresivo con las propias de una cultura del trabajo pragmática o instrumental; a partir de una sintesis que defienden la necesidad de posturas globales de desarrollo económico y social global para marcar al respecto la posición de los trabajadores. En ello, no solamente confluyen posiciones políticas, sino que también se avanza en una construcción cultural común y sin oposiciones internas que coloca al sindicato como una de las tribunas de los Derechos Humanos abriendo un espacio de reivindicación que trasciende la lucha tradicional contra el capitalismo, 
y que en este nuevo campo reivindicativo, encuentra tanto aquellos que tienen una orientación expresiva como aquellos que tienen una expresión pragmática o instrumental.

El enigma que se nos plantea en el marco de la investigación es: ¿cómo es posible que a través de la unidad sindical se mantenga la idea de la unidad de los trabajadores como clase, cuando el mundo de trabajo está crecientemente fraccionado ${ }^{11}$ ?

El sindicalismo se encuentra a nivel global con el hecho de que "clase obrera" pierde su hegemonía numérica en el seno del asalariado, pero también pierde la hegemonía como referente para una sociedad más justa a construir. Es en este marco que es necesario comprender la importancia del debate acerca de la unidad sindical y de las formas para alcanzarla.

El sindicalismo uruguayo tiene una unidad que lo diferencia de otros que cuentan con centrales que agrupan a los trabajadores a partir de distintas ideologías. Tampoco existe en Uruguay una distinción de centrales que asuman la representación de las distintas categorias laborales como se hace en muchos países de Europa. La unidad del sindicalismo uruguayo lograda en el PIT CNT es una unidad compleja que se transforma en el plano de las distintas perspectivas politicas existentes en su seno en una suerte de sistema politico específico, pero que no es un calco del sistema político uruguayo. Y en el plano de la variedad de trabajadores de distintas categorias que adhieren al sindicalismo constituye una identidad común que se manifiesta a partir de la unidad construida a nivel sindical. Esta identidad se fortalece a través del Congreso con la emotividad de los encuentros y de la votación a manos alzadas de las listas únicas para ocupar las instancias de mayor jerarquía del PIT CNT.

Ahora bien, esta unidad de tipo más politico que se ha logrado, ¿no supone previamente una cultura de unidad basada en el

\footnotetext{
11 La noción de trabajo y por extensión de trabajador, presupone una mayor cantidad de situaciones y acciones cada vez más disímiles
} 
El XIII Congreso del PIT CNT "Wladimir Turiansky" | Marcos Supervielle, Leonel Rivero \& Leonardo Cosse

reconocimiento de que el trabajo es una actividad colectiva que requiere fuertes actividades de coordinación, y que el lugar ocupado -de mayor o menor responsabilidad- en las organizaciones de trabajo es un aspecto coyuntural? Por lo tanto, la posibilidad de reagruparse tomando distancias de las categorías en las que los trabajadores están insertos, ¿no supone una cultura de trabajo altamente igualitaria en el cual la categorización de los trabajadores no suponga la constitución de cierto tipo de clases al interior del mundo del trabajo?

\section{Bibliografía}

ABRAMO, Laís y MONTERO Cecilia. Origen y evolución de la sociología del trabajo en América Latina. In: DE LA GARZA, Enrique. (coord..) Tratado de sociologia del Trabajo. Cidade do México: COLMEX, FLACSO, UAM, FCE, 2000.

BECKER, Howard. S. (2004) Epistémologie de la recherche qualitative> In: BLANC, Alain \& PESSIN, Alain. (coords.) L'Art du terrain. Paris: Ed L'Harmattan, 2004

BÉROUD, Sophie y DENIS, Jean - Michel. Les congrès syndicaux, un objet d'étude sociologique? In: Socio Logos Revue de l'association française d"etudes sociologiques, 2015,

LOURAU, René. El análisis institucional. Buenos Aires: Amorrortu, 1991.

MEDA, Dominique \& VENDRAMIN, Patricia. Reinventer le travail. Paris: PUF., 2013.

SUPERVIELLE, Marcos. Consideracões sobre Categorias do Entendimento revisitadas. In: ROBERTT, Pedro; RECH, Carla; LISDERO, Pedro \& FACHINETTO Rochelle Fellini (Orgs.) Metodologia em Ciências Sociais hoje: Práticas, abordagens e experiências de investigação. São Paulo: Paco Editorial, 2017. 